\title{
Dose Intensified Chemoradiation and Intraluminal Brachytherapy Improve Outcomes in Middle Third Carcinoma Esophagus: Experience from a Regional Cancer Center
}

\author{
Tanvirpasha C.R. ${ }^{1} \quad$ Siddanna R.P. ${ }^{1} \quad$ Bindu V. ${ }^{1} \quad$ Naveen T. ${ }^{1} \quad$ Lokesh V. ${ }^{1}$ \\ ${ }^{1}$ Department of Radiotherapy, Kidwai Memorial Institute of \\ Oncology, Bangalore, Karnataka, India

\begin{abstract}
Address for correspondence Siddanna R.P., MDRT, DNB, Department of Radiotherapy, Kidwai Memorial Institute of Oncology, Dr M H Marigowda
\end{abstract} \\ Road, Bangalore 560029, Karnataka, India (e-mail: siddannap@gmail.com).
}

\begin{abstract}
Keywords

- capecitabine

- carcinoma esophagus

- intraluminal

brachytherapy

Introduction Esophageal cancer is known for its poor outcome despite multimodality treatment. In this study, we report our experience with concurrent capecitabine-based chemoradiotherapy followed by intraluminal brachytherapy (ILBT) for middle third esophageal cancer.

Materials and Methods Twenty patients of histology-proven middle third esophageal cancer were treated with 45 Gray (Gy)/25 fraction ( $\mathrm{fr}$ ) of external beam radiation on telecobalt using two-dimensional technique with concurrent cisplatin $40 \mathrm{mg} / \mathrm{m}^{2}$ weekly with five such cycles along with tablet capecitabine $825 \mathrm{mg} / \mathrm{m}^{2}$ twice daily for the first 2 weeks and last 2 weeks of radiotherapy. This was followed by ILBT of $4 \mathrm{~Gy} \times 3 \mathrm{fr}$ placed 1 week apart from a total biologically effective dose of $60 \mathrm{~Gy}$. Patients were followed up with clinical examination and serial barium swallow to assess response and toxicity. Results Out of 20 patients, disease-free survival at 2 years was $60 \%$. At 5 years, the actuarial survival was $47 \%$ with five patients alive and two patients lost to follow-up at 3 years. One patient had grade 3 toxicity in the form of tracheoesophageal fistula (5\%). Two patients had distal failure. Two patients had second primary tumor in the upper aerodigestive tract at the end of 7 years.

Conclusion A combination of chemotherapy with cisplatin and capecitabine given concurrently with radical radiation therapy followed by ILBT boost is a safe and feasible protocol in the treatment of carcinoma esophagus involving middle third of esophagus. The local control and survival are comparable to historical studies with minimal toxicity.
\end{abstract}

\section{Introduction}

Esophageal cancer dominates the list of gastrointestinal (GI) malignancies with poor outcome. Despite multimodality treatment with surgery, radiation therapy (RT), and chemotherapy, the survival seems to be dismal. Most patients are at advanced stages of presentation and receive treatment with an intent of sustained palliation. Concurrent chemoradiation is the standard of care for radical, nonsurgical treatment with radiation alone reserved for palliation as established by Radiation Therapy Oncology Group (RTOG) 8501 study. ${ }^{1}$
Very often, these patients have multiple comorbidities and poor lung function resulting in inoperability or occasionally are unresectable due to poor response to neoadjuvant chemoradiation therapy. The onus therefore falls on the radiation oncologist to deliver doses capable of macroscopic cell kill. The patterns of failure in carcinoma esophagus patients postradical treatment with chemoradiation are local (50\%) out of which $90 \%$ is within the gross tumor ${ }^{2}$ the other $50 \%$ fails distally. Hence, dose escalation has been tried to achieve better local control that has not proven to be beneficial in
DOI https://doi.org/

$10.1055 / \mathrm{s}-0040-1708113$ ISSN 2454-6798.
License terms

()(1) $\Theta \circledast$ 
the INT 0123 trial. ${ }^{3,4}$ However, the mode of dose delivery was by external beam without conformity or dose modulation and the benefits of the increased dose may have been offset by tissue toxicity. Some more studies have shown increased locoregional control (69 vs. 32\%) and progression-free survival ( 47 vs. $20 \%)^{5}$ with increased RT doses to more than $>50$ Gray (Gy). Dose escalation can be achieved by Intraluminal brachytherapy (ILBT) taking advantage of the inverse square law to deliver highest dose to the mucosal surface of the esophagus with rapid dose fall off toward the lungs and other normal structures. Several studies have claimed that brachytherapy boost confers better symptom control, local control, and sustained palliation. ${ }^{6,7}$

There are various regimens of concurrent chemotherapy in carcinoma esophagus. The most common and time tested is one incorporating 5-fluorouracil (5-FU) with cisplatin. However, many centers have abandoned 5-FU and practice single-agent chemotherapy with cisplatin due to favorable toxicity profile. Capecitabine is a versatile drug with appropriate efficacy and minimal side effects compared with 5-FU. We conducted a prospective study examining the role of capecitabine in place of 5-FU with cisplatin for concurrent chemotherapy followed by ILBT for unresectable patients of middle third carcinoma esophagus.

\section{Objective}

The main aim of this study is to evaluate the safety and efficacy of concurrent capecitabine and cisplatin followed by ILBT in carcinoma esophagus patients with middle third involvement. This study also evaluates the outcomes following the treatment in terms of locoregional control, overall survival, and toxicity.

\section{Methodology}

A total of 20 patients of locally advanced and histologically proven carcinoma esophagus involving the middle third were chosen for the study. Patients included in the study were of either sex with a Karnofsky performance status of 80 or more and ECOG (Eastern Cooperative Oncology Group) performance status of 0 and 1, with histologically confirmed carcinoma esophagus. They underwent baseline investigations such as complete blood count, renal and liver function tests, and serology. Staging investigations included were barium swallow, computed tomography (CT) of thorax and abdomen, and bone scan where indicated. Endoscopic ultrasound was not used to stage due to unavailability. The lesions involving cervical esophagus and gastroesophageal junction were excluded from the study, as were distant metastatic lesions. Patients were also excluded from the study if they had any of the following: tumor length more than $8 \mathrm{~cm}$, tracheal infiltration or tracheoesophageal fistula (TOF) at presentation, stenosis which could not be negotiated, surgically operable at the end of external beam RT (EBRT) and out of field nodal involvement at presentation, as they would be unsuitable for brachytherapy boost as per American Brachytherapy Society guidelines. ${ }^{8}$ The AJCC 7th edition was used for staging the disease. ${ }^{9}$ Informed consent was obtained from all patients and Scientific and Ethical Committee clearance was obtained.

\section{Chemoradiation}

All patients were treated on telecobalt machine by two-dimensional technique with anteroposterior and posteroanterior beams to a dose of $45 \mathrm{~Gy}$ in 25 fractions (fr) at $1.8 \mathrm{~Gy}$ per fraction, five fractions per week. The volumes included were the gross tumor with a $5 \mathrm{~cm}$ craniocaudal margin and $1 \mathrm{~cm}$ radial margin to primary and nodal volume as appropriate.

All patients were planned to receive concurrent chemotherapy with injection cisplatin $40 \mathrm{mg} / \mathrm{m}^{2}$ weekly with five such cycles along with tablet capecitabine $825 \mathrm{mg} / \mathrm{m}^{2}$ twice daily for the first 2 weeks and last 2 weeks of radiotherapy. Chemotherapy was interrupted temporarily if the patient developed grade three hematological toxicity.

ILBT was planned by remote after-loading high-dose rate technique with GammaMed with Ir-192 source using ILBT applicator of $1 \mathrm{~cm}$ diameter after 1 to 2 weeks of EBRT. Patients were planned for three sessions with 4 Gy per fraction at $1 \mathrm{~cm}$ from the central axis planned using orthogonal X-rays, spaced 1 week apart amounting to a cumulative dose equivalent of $60 \mathrm{~Gy}$. The volumes treated were the gross tumor with $1 \mathrm{~cm}$ craniocaudal margin.

\section{Definition of Event}

All patients were examined weekly for reactions due to radiation and counts monitored for chemotherapy-related toxicity. Toxicity grading was done using the National Cancer Institute Common Toxicity Criteria version 3.

The patients were followed up posttreatment with clinical examination, endoscopy, barium swallow, and CT scans where indicated. No adjuvant chemotherapy was planned as part of the protocol. The treatment outcomes were tabulated in MS Excel and percentages were generated manually.

Complete response was defined as no evidence of disease on endoscopy or smooth flow of barium on a barium swallow. Partial response was defined as $>30 \%$ and $<100 \%$ response clinically and radiologically as per RECIST criteria version 1.1. Stable disease was defined as the response that does not qualify as PR or progressive disease.

Severe adverse events were recorded as grade 3 or more. Overall survival was defined as the survival from the first day of treatment to the last day of follow-up or date of death. Locoregional recurrence (LRC) was defined as recurrence within the field of radiation and recurrence outside of the field was considered distal recurrence.

All patients were advised to come for the first follow-up after 1 month and for every 3 months subsequently for up to 2 years, once in 6 months for up to 5 years, and annually thereafter.

\section{Results}

\section{Patient Characteristics}

There were 12 male patients and 8 female patients totally. The median age of all patients was 60 years. Seven patients were alcoholic, and nine patients were smokers. Seven patients 
were habituated to both alcohol and smoking. All cancers were squamous cell carcinomas. All patients received five cycles of cisplatin except one who received only one cycle.

\section{Response}

Complete response was seen in 10 patients, partial response in 9 patients, and stable disease in 1 patient.

\section{Survival}

The median follow-up time was 24 months. Out of 20 patients, disease-free survival at 2 years was $60 \%$. At 5 years, the actuarial survival was $47 \%$ with 5 patients alive and 2 patients lost to follow-up at 3 years. At 7 years ( $\boldsymbol{-}$ Figs. 1 and $\mathbf{2}$ ), 4 patients were on follow-up and alive.

\section{Toxicity}

The dysphagia reported during RT, immediately post-RT, and 3 months post-RT is given in - Table 1. Nine patients had a stricture at the end of 3 months. One patient developed TOF at the end of 6 months.

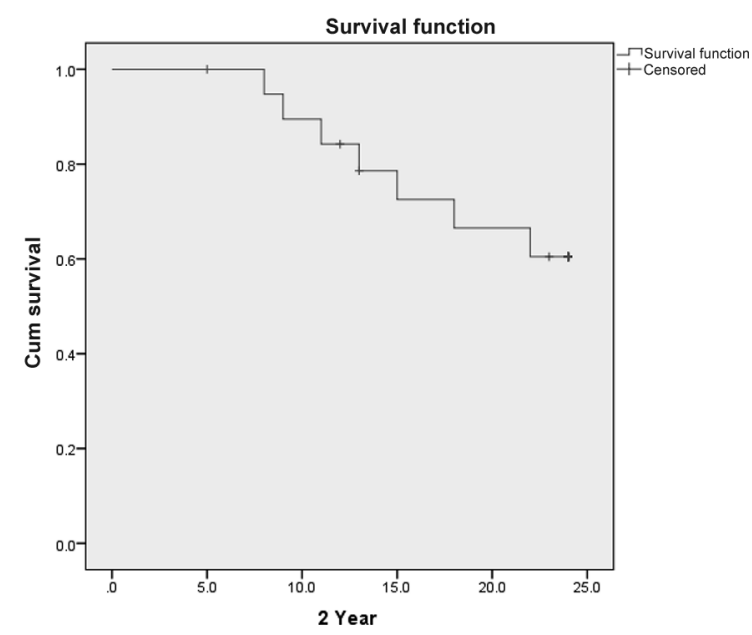

Fig. 1 Kaplan-Meier curve for 2-year survival postchemoradiation.

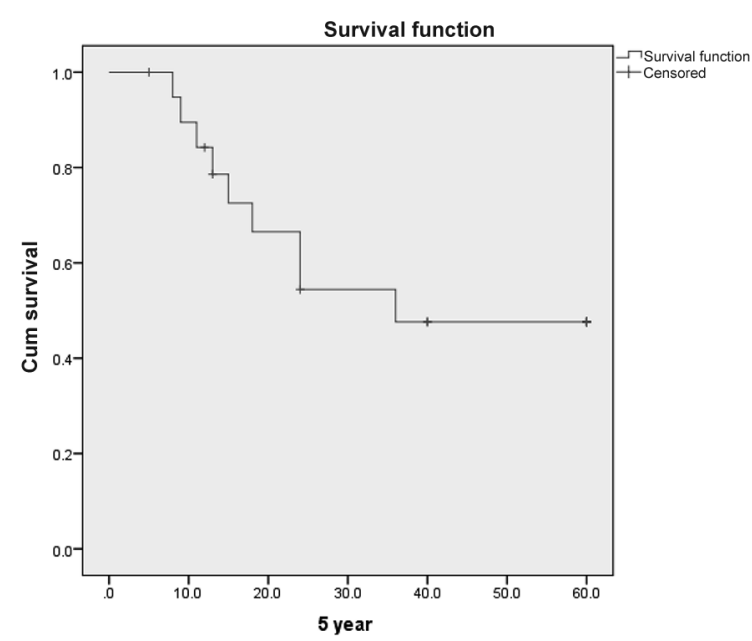

Fig. 2 Kaplan-Meier curve for 5-year survival postchemoradiation.

\section{Patterns of Failure}

One patient died from massive hemoptysis, possibly due to progressive disease at the end of 6 months. Out of the four patients alive at 7 years, two developed second primary tumor in the upper aerodigestive tract: one in the stomach and the other in the hypopharynx. Two patients had distant metastasis at the end of 1 year.

\section{Discussion}

The middle third esophageal cancer that is operable has traditionally been treated with radical surgery with or without adjuvant chemoradiation. RT has always played a key role in the treatment of carcinoma esophagus. The recommended dose of radiation with cytotoxic chemotherapy is $50.4 \mathrm{~Gy}$. However, it is observed that in operated cases of carcinoma esophagus, the pattern of recurrence is nodal or distal, whereas in definitive chemoradiation the site of failure is the primary. This has led to the hypothesis that at least in some patients it would be advantageous to increase the dose of radiation to the gross tumor in the setting of definitive treatment to improve LRC and hence survival. Middle third of esophagus is located ideally for dose escalation by ILBT since critical normal structures like trachea and the heart are relatively spared. Radiobiologically, the dose of $50 \mathrm{~Gy}$ is enough to take care of microscopic disease only. Hence dose escalation is required to achieve macroscopic cell kill.

ILBT has been used since the 1980s to palliate symptoms of dysphagia as well as in radical radiation as a boost. In our study, we observed that it contributed to improvement in locoregional control and overall survival. The rate of strictures reported in literature is $50 \%$ postchemoradiation in esophageal cancer. ${ }^{10}$ The factors predictive of occurrence of strictures include circumferential involvement of the disease, high grade of esophagitis during RT, stenosis at diagnosis, and endoscopic complete response. ${ }^{11,12}$ In this group of patients, all patients were managed with dilatation of strictures successfully. The most dreaded complication of ILBT is TOF which is reported to be as high as $17.5 \%$ posttreatment. ${ }^{10}$ In our present study, only one patient was diagnosed with TOF, that is, $5 \%$.

When radiation is given alone, the survival rate is seen to be less than $10 \%$ at 5 years. ${ }^{13}$ The intergroup trial RTOG 8501 has laid the foundation for the present day standard of care that incorporates concurrent chemotherapy. ${ }^{1}$ The intergroup trial incorporated cisplatin and 5-FU in the concurrent setting and established the benefit of these drugs in improving the 5-year survival rate from $0 \%$ with RT alone to $27 \%$ with chemoradiotherapy.$^{14}$ However, the practice of using 5-FU has seen a gradual withdrawal, especially in centers with poor supportive care infrastructure. The precursor of 5-FU and its oral counterpart capecitabine has been proposed as an alternative with favorable toxicity profile in many studies of the GI tract. This study confirms that 5-FU is an important component of chemotherapy and may be preferred in GI malignancies, either as a native injectable drug or in the form of capecitabine. 
Table 1 The toxicities graded according to CTC criteria in patients receiving chemoradiation

\begin{tabular}{|c|c|c|c|}
\hline Toxicity & During RT & One month post-RT & Three months post-RT \\
\hline Weight loss & 6 patients & - & - \\
\hline Weight gain & 10 patients & - & - \\
\hline Dysphagia & $\begin{array}{l}6 \text { (grade } 1) \\
13 \text { (grade } 2)\end{array}$ & $\begin{array}{l}14 \text { (grade } 1) \\
3 \text { (grade } 2)\end{array}$ & $\begin{array}{l}9 \text { (grade } 1) \\
4 \text { (grade } 2) \\
1 \text { (grade } 3)\end{array}$ \\
\hline Anemia & 6 (grade 1 ) & - & - \\
\hline Leucopenia & $\begin{array}{l}11 \text { (grade } 1 \text { ) } \\
2 \text { (grade } 2 \text { ) }\end{array}$ & - & - \\
\hline Neutropenia & $\begin{array}{l}11 \text { (grade } 1) \\
2 \text { (grade } 2 \text { ) }\end{array}$ & - & - \\
\hline Thrombocytopenia & 4 (grade 1 ) & - & - \\
\hline Stricture & - & 14 & 9 \\
\hline
\end{tabular}

Abbreviations: CTC, common toxicity criteria; RT, radiation therapy.

Capecitabine is a prodrug of 5-FU that can be administered orally. It has shown efficacy in various cancers such as colorectal, pancreatic, ovarian, and breast cancer. The advantages of capecitabine over 5-FU are the ease of administration and compliance, less hospital admissions for diarrhea, nausea and myelosuppression, and comparable efficacy. The present regimen was based on the use of capecitabine in other GI tumors like colorectal cancer that has shown equal efficacy to 5-FU with a reduction in the toxicity profile. ${ }^{15}$ Capecitabine is a reasonably good alternative to 5-FU; it is better tolerated and contributes to local disease control. Various regimens of capecitabine have been tried in GI malignancies. The regimen used in the protocol was well tolerated and there was no significant treatment interruptions with a median overall treatment time maintained to 54.5 days (range: 45-72 days).

\section{Conclusion}

A combination of chemotherapy with cisplatin and capecitabine given concurrently with radical RT followed by ILBT boost to a biologically effective dose of $60 \mathrm{~Gy}$ is a safe and feasible protocol in the treatment of carcinoma esophagus involving middle third of esophagus. Selection of patients for ILBT is of paramount importance to achieve maximal locoregional control while keeping the major toxicities at bay.

The toxicities resulting from this treatment are mainly esophageal strictures that can be managed conservatively. Grade three toxicities are better than that reported in literature. Further randomized studies need to be done to confirm these findings and to compare protocols involving surgery with radical chemoradiation.

\section{Conflict of Interest}

None declared.

\section{References}

1 Cooper JS, Guo MD, Herskovic A, et al; Radiation Therapy Oncology Group. Chemoradiotherapy of locally advanced esophageal cancer: long-term follow-up of a prospective randomized trial (RTOG 85-01) JAMA 1999;281(17):1623-1627
2 Welsh J, Settle SH, Amini A, et al. Failure patterns in patients with esophageal cancer treated with definitive chemoradiation. Cancer 2012;118(10):2632-2640

3 Minsky BD, Pajak TF, Ginsberg RJ, et al. INT 0123 (Radiation Therapy Oncology Group 94-05) phase III trial of combined-modality therapy for esophageal cancer: high-dose versus standard-dose radiation therapy. J Clin Oncol 2002;20(5):1167-1174

4 Minsky BD, Neuberg D, Kelsen DP, et al. Final report of Intergroup Trial 0122 (ECOG PE-289, RTOG 90-12): Phase II trial of neoadjuvant chemotherapy plus concurrent chemotherapy and high-dose radiation for squamous cell carcinoma of the esophagus. Int J Radiat Oncol Biol Phys 1999;43(3):517-523

5 Suh YG, Lee IJ, Koom WS, et al. High-dose versus standard-dose radiotherapy with concurrent chemotherapy in stages II-III esophageal cancer. Jpn J Clin Oncol 2014;44(6):534-540

6 Sur RK, Singh DP, Sharma SC, et al. Radiation therapy of esophageal cancer: role of high dose rate brachytherapy. Int J Radiat Oncol Biol Phys 1992;22(5):1043-1046

7 Okawa T, Dokiya T, Nishio M, Hishikawa Y, Morita K. Multi-institutional randomized trial of external radiotherapy with and without intraluminal brachytherapy for esophageal cancer in Japan. Int J Radiat Oncol Biol Phys 1999;45(3):623-628

8 Gaspar LE, Nag S, Herskovic A, Mantravadi R, Speiser B. American Brachytherapy Society (ABS) Consensus Guidelines for Brachytherapy in Esophageal Cancer. Clinical Research Committee, American Brachytherapy Society, Philadelphia, PA. Int J Radiat Oncol Biol Phys 1997;38(1):127-132

9 Edge SB, Byrd DR, Compton CC, et al. AJCC Cancer Staging Manual. 7th edn. New York, NY: Springer; 2010

10 Lepke RA, Libshitz HI. Radiation-induced injury of the esophagus. Radiology 1983;148(2):375-378

11 Uppal DK, Kang MS, Kaur T, et al. Predictive factors of esophageal stricture after definitive chemoradiation therapy for esophageal carcinoma: A retrospective single institute study. Medpulse Int Medical J 2019;9(3):174-178

12 Kim JW, Kim TH, Kim JH, Lee IJ. Predictors of post-treatment stenosis in cervical esophageal cancer undergoing high-dose radiotherapy. World J Gastroenterol 2018;24(7):862-869

13 Sun DR. Ten-year follow-up of esophageal cancer treated by radical radiation therapy: analysis of 869 patients. Int J Radiat Oncol Biol Phys 1989;16(2):329-334

14 al-Sarraf M, Martz K, Herskovic A, et al. Progress report of combined chemoradiotherapy versus radiotherapy alone in patients with esophageal cancer: an intergroup study. J Clin Oncol 1997;15(1):277-284

15 Walko CM, Lindley C. Capecitabine: a review. Clin Ther $2005 ; 27(1): 23-44$ 